\title{
High spatial resolution hard X-ray microscope using X-ray refractive lens and phase contrast imaging experiments
}

\author{
Y. Kohmura ${ }^{\mathrm{a} *}$, K. Okada ${ }^{\mathrm{a}}$, A. Takeuchi ${ }^{\mathrm{a}}$, H. Takano ${ }^{\mathrm{a}}$, Y. Suzuki ${ }^{\mathrm{a}}$, T. Ishikawa ${ }^{\mathrm{a}}$, \\ T. Ohigashi ${ }^{\mathrm{b}}$, H. Yokosuka ${ }^{\mathrm{b}}$ \\ ${ }^{a}$ SPring-8, 1-1-1 Kouto, Mikazuki-cho, Sayo-gun, Hyogo 679-5143, Japan \\ ${ }^{\mathrm{b}}$ Institute of Applied Physics, University of Tsukuba, Tsukuba, Ibaraki 305, Japan
}

\begin{abstract}
A high spatial resolution X-ray microscope was constructed using an X-ray refractive lens as an objective. The spatial resolution was tested using $18 \mathrm{keV}$ X-ray. A $0.4 \mu \mathrm{m}$ line and $0.4 \mu \mathrm{m}$ space tantalum test pattern was successfully resolved. Using the similar setup with the addition of a phase plate, a Zernike type phase-contrast microscopy experiment was carried out for the phase retrieval of the samples. Two-dimensional phase-contrast images were successfully taken for the first time in the hard X-ray region. Images of a gold mesh sample were analyzed and the validity of this method was indicated. An improvement of the lens, however, is required for the precise phase retrieval of the samples. (C) 2001 Elsevier Science B.V. All rights reserved.
\end{abstract}

PACS: $07.85 . \mathrm{Y} ; 41.50$

Keywords: X-ray refractive lens; Spatial resolution; Application; Synchrotron radiation

\section{Introduction}

X-ray refractive lenses are attractive for focusing and imaging experiments at the hard X-ray region. Unlike diffractive optical elements, higher order foci are not present, therefore the alignment is simple and a low background can be achieved $[1,2]$. Raytrace simulations are easy for optimizing the experiments [3,4]. The microscope system

\footnotetext{
*Corresponding author. Harima Institute, RIKEN (The Institute of Physical and Chemical Research), 1-1-1 Kouto, Mikazuki, Sayo, Hyogo 679-5148, Japan. Tel.: +81-791-582806; fax: +81-791-58-2807.

E-mail address: kohmura@sp8sun.spring8.or.jp (Y. Kohmura).
}

reported here used a micro-capillary X-ray refractive lens $[3,4]$ as an objective. The spatial resolution tested at $18 \mathrm{keV}$ was about $0.4 \mu \mathrm{m}$ as described later. Phase-sensitive imaging by the Zernike-type phase-contrast method is also reported.

\section{Experimental setup and evaluation of spatial resolution}

The hard X-ray microscope reported here was composed of a micro-capillary X-ray refractive lens [3,4], made of epoxy glue with $\sim 185$ air bubbles. The evaluation of the spatial resolution 
was done at BL47XU of SPring-8, a standard undulator beamline with a cryogenically cooled silicon double-crystal monochromator. The focal length of the X-ray refractive lens was $f \sim 0.48 \mathrm{~m}$ at $18 \mathrm{keV}$. The transmission image of a sample (a tantalum resolution test pattern) is magnified with the lens by a factor of 10 . The two-dimensional detector was composed of a fluorescence screen and a charge coupled device (CCD) camera with a pixel size of $6 \mu \mathrm{m}$ and a minimum exposure time of $20 \mathrm{~ms}$.

The tantalum test pattern was fabricated by NTT-AT. Corp., Japan using the reactive-ionetching method, coating tantalum $(0.5 \mu \mathrm{m}$ in thickness) on a $\mathrm{Si}_{3} \mathrm{~N}_{4}$ membrane $(2 \mu \mathrm{m}$ in thickness).

The recorded X-ray microscopy image is shown in Fig. 1. As shown by arrows in Fig. 1, the $0.4 \mu \mathrm{m}$ line and $0.4 \mu \mathrm{m}$ space stripe pattern was clearly

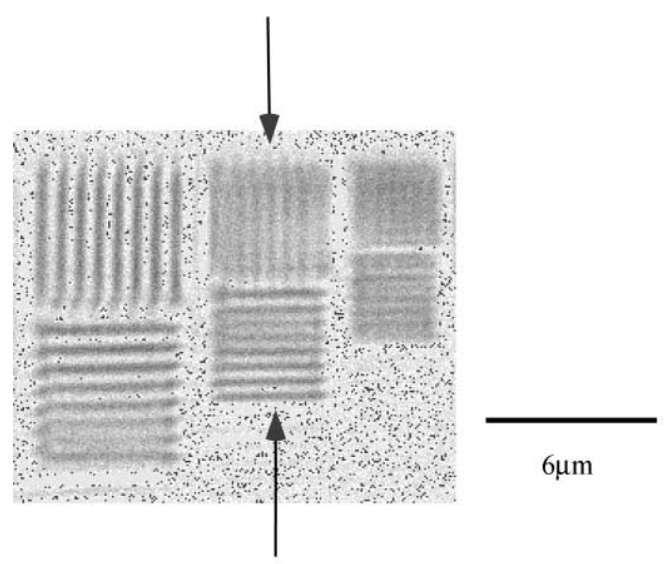

Fig. 1. The transmission image of the tantalum test pattern $(0.5 \mu \mathrm{m}$ in thickness) using the $18 \mathrm{keV} \mathrm{X}$-rays. Eight lines were resolved at the $0.4 \mu \mathrm{m}$ line and $0.4 \mu \mathrm{m}$ space stripe pattern (see arrows). resolved, in spite of the high transmissivity of $\sim 94 \%$ through the coated tantalum.

\section{Zernike-type phase contrast microscopy}

A Zernike-type phase-contrast microscopy experiment was carried out at BL20B2, a standard bending magnet beamline of SPring- 8 . The focal length of the X-ray lens was $f \sim 0.85 \mathrm{~m}$ at $24 \mathrm{keV}$. This long beamline, $200 \mathrm{~m}$ in total, enables us to design a system with an extremely large magnification factor $(M \sim 190)$ (Fig. 2).

A gold $\lambda / 4$ phase plate was installed at the backfocal plane of the lens to advance the phase of the undeflected X-rays (0th order X-rays) from the sample. The utilized phase plate was fabricated by Nikon corp., Japan. The thickness was chosen to be $2.3 \mu \mathrm{m}$, to precisely give $\pi / 2$ phase shift for the $24 \mathrm{keV} \mathrm{X}$-rays and the shape of this phase plate was a circle of $20 \mu \mathrm{m}$ in diameter.

The result of the observation of a gold \#1500 mesh (purchased from Good Fellow corp., Britain) is reported here. The mesh pitch, the wire width and the thickness were 16.7, 5.6 and $\sim 5 \mu \mathrm{m}$, respectively. The phase-shift and the transmissivity through the gold wire were about $1.2 \pi$ and $63 \%$ at $24 \mathrm{keV}$. Fig. 3(a) shows the phase-contrast image with a phase plate in the beam.

The computer simulations of the images were made for the obtained image with the following assumptions. (i) A parallel incident X-ray beam and an aberration-free lens were assumed. (ii) The pitch, width and thickness of the gold wires were assumed to be $16.7,5.6$ and $\sim 5 \mu \mathrm{m}$, respectively. (iii) The cross-section of the gold wire was assumed to be a square with a uniform thickness of $5 \mu \mathrm{m}$.

The simulation was carried out as follows. (i) The complex Fourier transformation was made on the amplitude and phase distribution at the

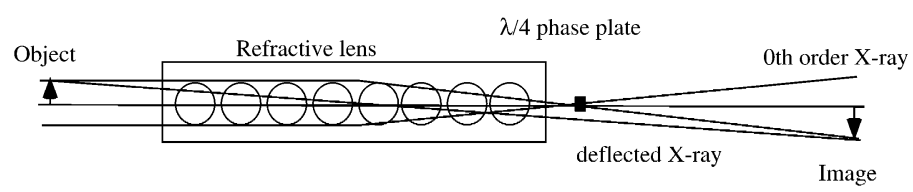

Fig. 2. Setup for the Zernike-type phase-contrast microscope at BL20B2 of SPring-8 (by setting the distances to $L_{1}=0.85 \mathrm{~m}$ and $L_{2}=160 \mathrm{~m}$, respectively), where $L_{1}$ and $L_{2}$ represent distance from sample to lens and distance from lens to detector, respectively. 

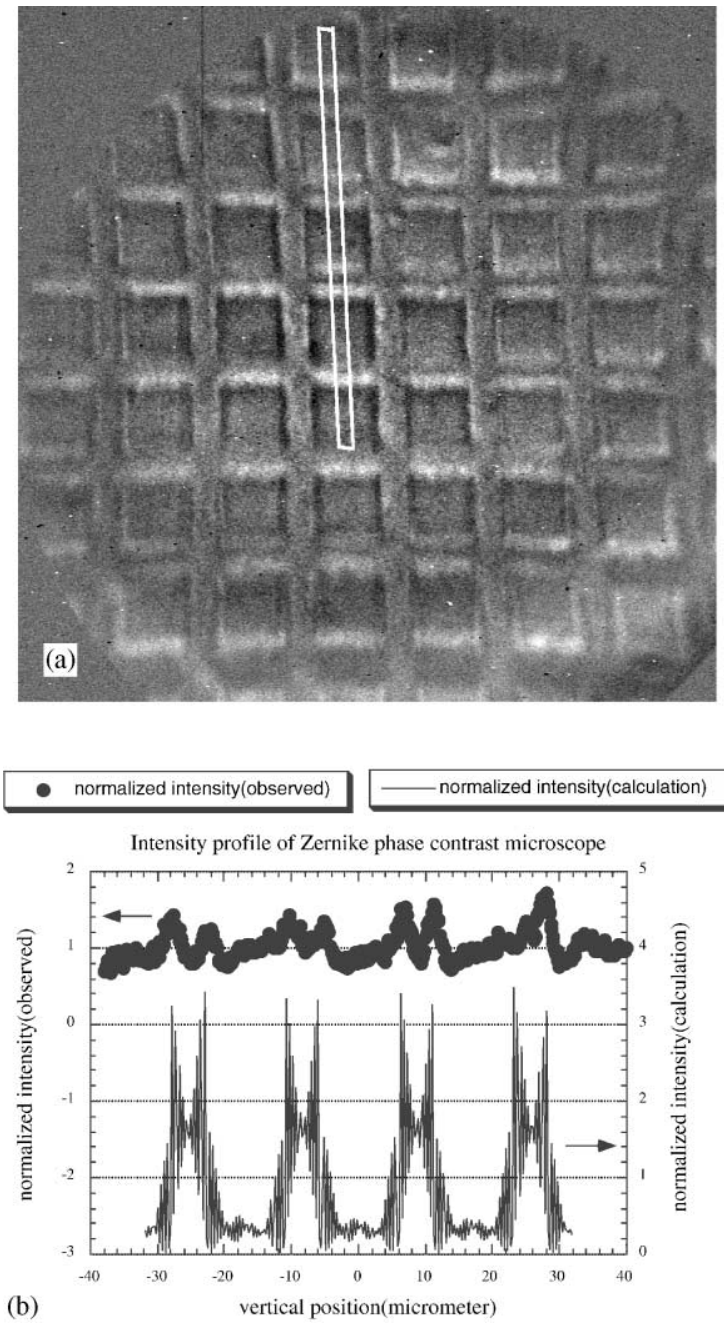

Fig. 3. The images of the gold \#1500 mesh at $24 \mathrm{keV}$. (a) Phasecontrast image with phase plate in the beam. (b) The vertical projection of the area enclosed by a square in (a) and the calculated profile. The details of the calculation are described in the text.

back surface of the sample so that the angular distribution at the back-focal plane of the lens can be calculated. (ii) The phase advance of $\pi / 2$ was made for the angular components $(|\Delta \theta| \leqq 11.8 \mu \mathrm{rad}$ from the optical axis, due to the finite radius of the phase plate). Finally, (iii) an inverse Fourier transformation was made.

Vertical intensity profile of the observed image shows double peaks at the edges of the gold wires which are also observed in the simulated intensity profile (Fig. 3(b)). The projection of the data was normalized by the X-ray-beam-intensity after the subtraction of the detector dark-noise. The calculated profile, on the other hand, was normalized by the intensity without any sample. A flat intensity distribution and a parallel incident X-ray were assumed in the simulation. The observed image contrast (peak/valley) was much lower than the calculated profile, probably due to the aberrations of the lens and the partial coherence of the X-ray beam.

\section{Summary}

An X-ray microscope system with a high spatial resolution was successfully constructed using a micro-capillary X-ray refractive lens as an objective. A $0.4 \mu \mathrm{m}$ line and $0.4 \mu \mathrm{m}$ space tantalum test pattern was successfully resolved using this system at $18 \mathrm{keV}$.

Zernike type phase-contrast microscopy experiment was carried out for the phase retrieval of the samples. Two-dimensional Zernike type phasecontrast images were successfully taken for the first time in the hard X-ray region. The simulation qualitatively reproduced the observed vertical and horizontal intensity profiles. This showed the validity of this method. The image contrast, however, was much lower than the calculation probably due to the aberrations and imperfections of the lens. Therefore, an improvement of the lens is needed.

For improving the image contrast, a parabolic lens with reduced aberration is under development by precise drilling on aluminum plates.

\section{References}

[1] A. Snigirev, V. Kohn, I. Snigireva, A. Souvorov, B. Lengeler, Appl. Opt. 37 (1998) 653.

[2] B. Lengeler, J. Tummer, A. Snigirev, I. Snigireva, C. Raven, J. Appl. Phys. 84 (1998) 5855.

[3] Y. Kohmura, M. Awaji, Y. Suzuki, T. Ishikawa, Y.I. Dudchik, N.N. Kolchevsky, F.F. Komarov, Rev. Sci. Instrum. 70 (1999) 4164.

[4] Y.I. Dudchik, N.N. Kolchevsky, Nucl. Instr. Meth. Phys. Res. A 421 (1999) 361. 